\title{
PROOF OF THE WELL-ORDERING \\ OF CARDINAL NUMBERS
}

\author{
CHAIM SAMUEL HÖNIG
}

It is well known that the class of cardinal numbers is well-ordered. But the proofs that we know are long ones, using Zorn's Theorem and the cumbersome theory of ordinal numbers. In this paper we give a very short proof of this theorem using both the Axiom of Choice and Zorn's Theorem.

By the theorem of Bernstein-Cantor we know that the cardinal numbers form an order class. If we prove that every family of cardinal numbers has a first element it will follow that it is totally ordered (if not, a set of two incomparable elements would not have a first element) and indeed, well-ordered.

We shall use the notations and terminology of Bourbaki.

Theorem 1. Let $\left(\boldsymbol{\aleph}_{i}\right)_{i \in I}$ be a family of cardinal numbers of subsets $A_{i}$ of a set $E$. Then there exists an $i_{0} \in I$ such that $\boldsymbol{\aleph}_{i_{0}} \leqq \boldsymbol{\aleph}_{i}$ for every $i \in I$.

Proof. We shall have our result if we prove that for every $i \in I$ there exists a one-to-one function $f_{i}$ from $A_{i_{0}}$ in to $A_{i}$.

Let us take the cartesian product $A=\prod_{i \in I} A_{i}$ and let us consider the class $B$ of subsets $B$ of $A$ such that

$\left(^{*}\right) \quad x=\left(x_{i}\right) \in B, y=\left(y_{i}\right) \in B$, and $x \neq y$ implies $x_{i} \neq y_{i}$ for every $i \in I^{*}$

It is immediate that $B$ is inductive when ordered by inclusion and hence, by Zorn's Theorem, $B$ has at least one maximal element $\bar{B}$. We assert that there exists an $i_{0} \in I$ such that $\operatorname{pr}_{i_{0}}(\bar{B})=A_{i_{0}}$ : for if $p r_{i}(\bar{B}) \neq A_{i}$ for every $i \in I$ we could take (by the Axiom of Choice) an element $a_{i}$ in every $A_{i}-\operatorname{pr}_{i}(\bar{B})$ and set $\bar{B} \cup\{a\}$ where $a=\left(a_{i}\right)$ would strictly contain $\bar{B}$ and it would still satisfy $\left({ }^{*}\right)$ and so $\bar{B}$ would not be maximal. If $p r_{i_{0}}(\bar{B})=A_{i_{0}}$ we define the one-to-one function $f_{i}$ from $A_{i_{0}}$ into $A_{i}$ by $x_{i_{0}} \in A_{i_{0}} \rightarrow f_{i}\left(x_{i_{0}}\right)=x_{i}=p r_{i} x$ where $x$ is the point of $\bar{B}$ such that $p r_{i_{0}} x=x_{i_{0}}$; by (*) this point $x \in \bar{B}$ is unique and $f_{i}$ is one-toone $\left(x_{i_{0}} \neq y_{i_{0}} \rightarrow x \neq y \rightarrow x_{i} \neq y_{i}\right)$. Q.E.D.

CoRollary. The cardinal numbers of subsets of a set $E$ form a wellordered set.

University of São Paulo

Received by the editors June 28, 1953. 